\title{
MHD Mixed Convection Flow of a Micropolar Fluid Past a Wedge Fixed in a Fluctuating Free Stream and Surface Temperature
}

\author{
N. C. Roy* , P. Akther and A. K. Halder \\ Department of Mathematics, Dhaka University, Dhaka-1000, Bangladesh
}

(Received: 9 July 2015; Accepted: 29 November 2015)

\begin{abstract}
The flow and heat transfer on the unsteady laminar mixed convection boundary layer in a micropolar fluid past a vertical wedge have been studied taking into account the effect of magnetic field. We assume that the free stream velocity and surface temperature oscillate in magnitude but not in the direction of the oncoming flow velocity. The governing equations have been solved numerically by using the straight forward finite difference method. The amplitudes of skin friction and couple stress are found to be significantly dependent on the Richardson's number, $R i$, the magnetic parameter, $M$, and the vortex viscosity parameter, $K$. We observe that the amplitudes of skin friction and couple stress increases owing to an increase of the Richardson's number, $R i$, while these become lower for the higher value of the magnetic parameter, $M$, and the vortex viscosity parameter, $K$. Also the results demonstrate that the effects of the parameters on the amplitudes of heat transfer are rather weak.
\end{abstract}

\section{Introduction}

Over decades, micropolar fluids have been the focus of research of many researchers working in the field of fluid mechanics owing to its practical applications in many industrial and engineering applications. Micropolar fluids are fluids with microstructure and asymmetrical stress tensor. These fluids consist of rigid, randomly oriented or spherical particles with their own spins and micro-rotations, suspended in a viscous medium. Eringen ${ }^{1,2}$ formulated the theory of micropolar fluids that elucidates the effect arising from the local structure and micro-motions of the fluid elements. A comprehensive review of this subject and application of micropolar fluid mechanics has been accomplished by Ariman et al. ${ }^{3}$. The characteristics of the boundary layer flow and heat transfer of micropolar fluids are of technological importance and so it has been studied under a variety of physical conditions.

The concept of boundary layer in micropolar fluids was first introduced by Willson ${ }^{4}$. He studied the steady, incompressible laminar flow over two-dimensional bodies. Later, Peddieson and $\mathrm{McNitt}^{5}$ extended this to investigate the steady boundary layer flow of micropolar fluids at the stagnation point of a two-dimensional body. In addition to, Ahmadi ${ }^{6}$ considered the steady boundary layer flow of micropolar fluids over a semi-infinite flat plate and obtained a self-similar solution. Ramachandran et al. ${ }^{7}$ and Gorla ${ }^{8}$ studied the thermal boundary layer in micropolar fluids at the stagnation point of a two-dimensional body. Gorla ${ }^{9,10}$ also investigated the boundary layer characteristics of an axisymmetric, laminar, micropolar fluid flow along a horizontal cylinder using the theory of micropolar fluids formulated by Eringen ${ }^{1,2}$. On the other hand, Rees and Bassom $^{11}$ examined the Blasius boundary-layer flow of a micropolar fluid over a flat plate. They derived nonsimilar boundary layer equations that were solved by the Keller-box method. They also performed an asymptotic analysis for large distances from the leading edge because the numerical results indicate that the boundary layer develops a two-layer structure.
Due to the growing demand of technological appliances, there are many cases in which magnetic fields are strongly encountered in an electrically conducting fluid, for example, electric power generation, astrophysical flows, solar power technology, space vehicle re-entry, nuclear engineering applications, etc. Magnetohydrodynamic (MHD) mixed convection heat transfer flow is of practical importance owing to its frequent occurrence in industrial technology and geothermal applications, high temperature plasmas applicable to nuclear fusion energy conversion, liquid metal fluids, and (MHD) power generation systems. Ishak ${ }^{12}$ examined the MHD boundary layer flow of a micropolar fluid past a wedge with constant wall heat flux. An exact solution for unsteady magnetohydrodynamic free-convection flow over an impulsively moving vertical plate with constant heat flux was given by Sacheti et al. ${ }^{13}$ and the results show that the magnetic field has a retarding effect on the velocity profiles while the surface skin friction increases with it. Ibrahim et al. ${ }^{14}$ obtained the analytical solution for unsteady MHD free convection flow past a semi-infinite vertical permeable moving plate with heat source and chemical reaction.

The objective of this study is to investigate the flow and heat transfer characteristics on the unsteady laminar mixed convection boundary layer in a micropolar fluid past a vertical wedge. Results have been demonstrated in terms of the amplitudes of the skin friction, couple stress and heat transfer with the variations of the Richardson's number, $R i$, vortex viscosity parameter, $K$ and magnetic parameter, $M$.

\section{Mathematical Formalisms}

A two-dimensional, unsteady, laminar mixed convection flow of an incompressible micropolar fluid flow past a wedge is considered. The flow configuration and coordinate system are shown in Figure 1. Under the usual boundary layer approximation, the governing equations of conservation of momentum, angular momentum and energy in the presence of magnetic field can be expressed as:

$$
\frac{\partial u}{\partial x}+\frac{\partial v}{\partial y}=0
$$

\footnotetext{
*Author for correspondence. e-mail: nepal@du.ac.bd
} 


$$
\begin{aligned}
& \frac{\partial u}{\partial t}+u \frac{\partial u}{\partial x}+v \frac{\partial u}{\partial y} \\
& =\frac{\partial U}{\partial t}+U \frac{\partial U}{\partial x}+\frac{\mu+\kappa}{\rho} \frac{\partial^{2} u}{\partial y^{2}}+\frac{\kappa}{\rho} \frac{\partial N}{\partial y} \\
& +g \beta\left(T-T_{\infty}\right) \cos \left(\frac{\pi}{4}\right)-\frac{\sigma B_{0}^{2}}{\rho} u, \\
& \frac{\partial N}{\partial t}+u \frac{\partial N}{\partial x}+v \frac{\partial N}{\partial y}=\frac{\gamma}{\rho j} \frac{\partial^{2} N}{\partial y^{2}} \\
& -\frac{\kappa}{\rho j}\left(2 N+\frac{\partial u}{\partial y}\right), \\
& \frac{\partial T}{\partial t}+u \frac{\partial T}{\partial x}+v \frac{\partial T}{\partial y}=\alpha \nabla^{2} T .
\end{aligned}
$$

Here $x$ and $y$ are the coordinates parallel with and perpendicular to the wedge surface, $u$ and $v$ are the velocity components, $T$ is the fluid temperature, $T_{\infty}$ is the temperature of the ambient fluid, $\rho$ is the density, $\alpha$ is the thermal diffusivity, $j$ is the micro inertia density, $\mu$ is the coefficient of viscosity, $\kappa$ is the coefficient of gyroviscosity (or vortex viscosity), $\sigma$ is the electrical conductivity, $g$ is the acceleration due to gravity, $\beta$ is the coefficient of volumetric expansion, $N$ is the component of the micro-rotation vector normal to the $x y$-plane, $B_{0}$ is the strength of magnetic field which is assumed to be applied in the positive $y$ direction, normal to the surface. We have considered that $B_{0}$ is proportional to $x^{-(1-m) / 2}$, that is, the magnetic field is strong near the leading edge and weak in the downstream direction.

Furthermore, the spin-gradient viscosity $\gamma$, which gives some relationship between the coefficients of viscosity and micro-inertia, is defined as

$$
\gamma=\left(\mu+\frac{\kappa}{2}\right) j
$$

The above governing equations need to be solved subject to the following boundary conditions on velocity, microrotation and temperature fields:

$$
\begin{aligned}
& u=v=0, \quad N=-\frac{1}{2} \frac{\partial u}{\partial y}, \\
& T=T_{\infty}+\left(T_{w}-T_{\infty}\right)\left(1+\varepsilon e^{i \omega t}\right) \text { at } y=0 \\
& u=U(x, t), \quad v=0, \quad N=0, \\
& T=T_{\infty} \text { as } y \rightarrow \infty .
\end{aligned}
$$

where $T_{w}$ is the surface temperature of the wedge. The boundary condition (6) means that the micro-rotation is equal to half of the fluid vorticity at the boundary.

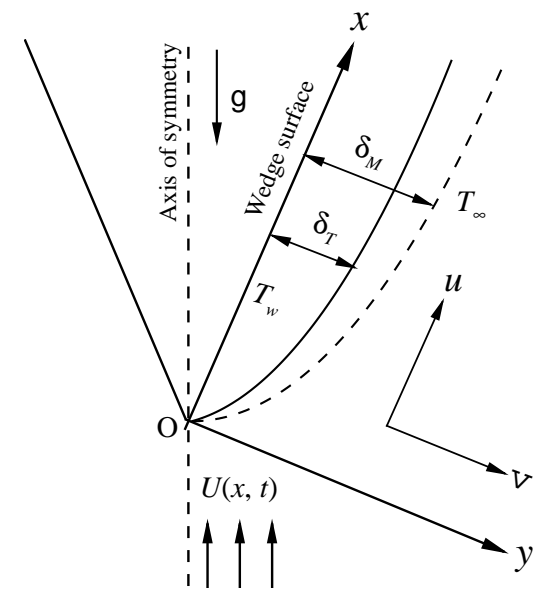

Fig. 1. Flow configuration and coordinate system

Here, the free-stream velocity $U(x, t)$ is assumed to be of the form

$U(x, t)=U_{0} x^{m}\left(1+\varepsilon e^{i \omega t}\right)$,

where $U_{0}, \varepsilon$ are constants and $\omega$ is the frequency.

The equation of continuity (1) is satisfied if we choose the stream function $\psi$ such that

$\bar{u}=\frac{\partial \psi}{\partial \bar{y}}, \quad \bar{v}=-\frac{\partial \psi}{\partial \bar{x}}$

Since, the amplitudes of the free-stream velocity and temperature variation $\varepsilon(<<1)$ is very small, we now assume the solutions of the following forms

$$
\begin{aligned}
& \psi(x, y, t)=\psi_{s}(x, y)+\varepsilon \psi_{1}(x, y) e^{i \omega t}, \\
& N(x, y, t)=N_{s}(x, y)+\varepsilon N_{1}(x, y) e^{i \omega t}, \\
& T(x, y, t)=T_{s}(x, y)+\varepsilon T_{1}(x, y) e^{i \omega t},
\end{aligned}
$$

where only the real part is to be taken as it has physical meaning.

In order to obtain the steady state solutions, we substitute (9)-(10) into (2)-(4), and equate the coefficients of $\varepsilon^{0}$ to obtain

$$
\begin{aligned}
& \frac{\partial \psi_{s}}{\partial y} \frac{\partial^{2} \psi_{s}}{\partial x \partial y}-\frac{\partial \psi_{s}}{\partial x} \frac{\partial^{2} \psi_{s}}{\partial y^{2}} \\
& =m U_{0}^{2} x^{2 m-1}+\frac{\mu+\kappa}{\rho} \frac{\partial^{3} \psi_{s}}{\partial y^{3}}+\frac{\kappa}{\rho} \frac{\partial N_{s}}{\partial y} \\
& +g \beta_{T}\left(T_{s}-T_{\infty}\right)-\frac{\sigma B_{0}^{2}}{\rho} \frac{\partial \psi_{s}}{\partial y},
\end{aligned}
$$

$\frac{\partial \psi_{s}}{\partial y} \frac{\partial N_{s}}{\partial x}-\frac{\partial \psi_{s}}{\partial x} \frac{\partial N_{s}}{\partial y}=\frac{\gamma}{\rho j} \frac{\partial^{2} N_{s}}{\partial y^{2}}$

$-\frac{\kappa}{\rho j}\left(2 N_{s}+\frac{\partial^{2} \psi_{s}}{\partial y^{2}}\right)$, 
$\frac{\partial \psi_{s}}{\partial y} \frac{\partial T_{s}}{\partial x}-\frac{\partial \psi_{s}}{\partial x} \frac{\partial T_{s}}{\partial y}=\alpha \frac{\partial^{2} T_{s}}{\partial y^{2}}$.

The set of equations for $\psi_{s}, N_{s}$ and $T_{s}$ represents the steadystate solutions that can be determined by the following functions:

$\psi_{s}=\left(v U_{0}\right)^{\frac{1}{2}} x^{(m+1) / 2} f(\eta)$,

$N_{s}=U_{0}\left(U_{0} / v\right)^{1 / 2} x^{(3 m-1) / 2} h(\eta)$,

$T_{s}=T_{\infty}+\left(T_{w}-T_{\infty}\right) \theta(\eta)$,

$\eta=\left(U_{0} / v\right)^{1 / 2} x^{(m-1) / 2} y, j=\left(v / U_{0}\right) x^{1-m}$.

Using (14) in (11)-(13), we obtain the dimensionless equations

$$
\begin{aligned}
& (1+K) f^{\prime \prime \prime}+K h^{\prime}+\frac{m+1}{2} f f^{\prime \prime} \\
& +m\left(1-f^{\prime 2}\right)+R i \theta-M f^{\prime}=0, \\
& \left(1+\frac{K}{2}\right) h^{\prime \prime}+\frac{m+1}{2} f h^{\prime}-K\left(2 h+f^{\prime \prime}\right)=0, \\
& \frac{1}{\operatorname{Pr}} \theta^{\prime \prime}+\frac{m+1}{2} f \theta^{\prime}=0 .
\end{aligned}
$$

The modified boundary conditions are

$$
f=f^{\prime}=0, h=-\frac{1}{2} f^{\prime \prime}, \theta=1 \text { at } \eta=0,
$$

$f^{\prime}=1, h=0, \theta=0$ as $\eta \rightarrow \infty$.

Invoking the expressions (8)-(10) into (2)-(4), and equating the coefficients of $\varepsilon$ we obtain time-dependent components $\psi_{1}, N_{1}$ and $T_{1}$ as

$i \omega \frac{\partial \psi_{1}}{\partial y}+\frac{\partial \psi_{s}}{\partial y} \frac{\partial^{2} \psi_{1}}{\partial x \partial y}+\frac{\partial \psi_{1}}{\partial y} \frac{\partial^{2} \psi_{s}}{\partial x \partial y}$ $-\frac{\partial \psi_{s}}{\partial x} \frac{\partial^{2} \psi_{1}}{\partial y^{2}}-\frac{\partial \psi_{1}}{\partial x} \frac{\partial^{2} \psi_{s}}{\partial y^{2}}=i \omega U_{0} x^{m}$

$+2 m U_{0}^{2} x^{2 m-1}+\frac{\mu+\kappa}{\rho} \frac{\partial^{3} \psi_{1}}{\partial y^{3}}+\frac{\kappa}{\rho} \frac{\partial N_{1}}{\partial y}$

$+g \beta T_{1}-\frac{\sigma B_{0}^{2}}{\rho} \frac{\partial \psi_{1}}{\partial y}$,

$i \omega N_{1}+\frac{\partial \psi_{s}}{\partial y} \frac{\partial N_{1}}{\partial x}+\frac{\partial \psi_{1}}{\partial y} \frac{\partial N_{s}}{\partial x}$

$-\frac{\partial \psi_{s}}{\partial x} \frac{\partial N_{1}}{\partial y}-\frac{\partial \psi_{1}}{\partial x} \frac{\partial N_{s}}{\partial y}=\frac{\gamma}{\rho j} \frac{\partial^{2} N_{1}}{\partial y^{2}}$

$-\frac{\kappa}{\rho j}\left(2 N_{1}+\frac{\partial^{2} \psi_{1}}{\partial y^{2}}\right)$

$i \omega T_{1}+\frac{\partial \psi_{s}}{\partial y} \frac{\partial T_{1}}{\partial x}+\frac{\partial \psi_{1}}{\partial y} \frac{\partial T_{s}}{\partial x}-\frac{\partial \psi_{s}}{\partial x} \frac{\partial T_{1}}{\partial y}$

$-\frac{\partial \psi_{1}}{\partial x} \frac{\partial T_{s}}{\partial y}=\alpha \frac{\partial^{2} T_{1}}{\partial y^{2}}$.
The transformed boundary conditions become

$$
\begin{aligned}
& \psi_{1}=\frac{\partial \psi_{1}}{\partial y}=0, \quad N_{1}=-\frac{1}{2} \frac{\partial^{2} \psi_{1}}{\partial y^{2}}, \\
& T_{1}=T_{w}-T_{\infty} \text { at } y=0, \\
& \frac{\partial \psi_{1}}{\partial \bar{y}}=U_{0} x^{m}, N_{1} \rightarrow 0, T_{1} \rightarrow 0 \text { as } y \rightarrow \infty .
\end{aligned}
$$

To non-dimensionalize the equations (20), (21), and (22), we introduce the following expressions

$$
\begin{aligned}
& \psi_{1}=\left(v U_{0}\right)^{\frac{1}{2}} x^{(m+1) / 2} F(\xi, \eta), \\
& N_{1}=U_{0}\left(U_{0} / v\right)^{1 / 2} x^{(3 m-1) / 2} H(\xi, \eta), \\
& T_{1}=\left(T_{w}-T_{\infty}\right) \Theta(\xi, \eta) .
\end{aligned}
$$

Using (14) and (25) into (20)-(24), we obtain

$$
\begin{aligned}
& (1+K) F^{\prime \prime \prime}+\frac{m+1}{2}\left(f F^{\prime \prime}+F f^{\prime \prime}\right)+i \xi\left(1-F^{\prime}\right) \\
& +2 m\left(1-f^{\prime} F^{\prime}\right)-M F^{\prime}+K H^{\prime}+R i \Theta \\
& =(1-m) \xi\left(f^{\prime} \frac{\partial F^{\prime}}{\partial \xi}-f^{\prime \prime} \frac{\partial F}{\partial \xi}\right), \\
& \left(1+\frac{K}{2}\right) H^{\prime \prime}+\frac{m+1}{2}\left(f H^{\prime}+F h^{\prime}\right)-i \xi H \\
& -K\left(2 H+F^{\prime \prime}\right)=(1-m) \xi\left(f^{\prime} \frac{\partial H}{\partial \xi}-h^{\prime} \frac{\partial F}{\partial \xi}\right), \\
& \frac{1}{\operatorname{Pr}} \Theta^{\prime \prime}+\frac{m+1}{2}\left(f \Theta^{\prime}+F \theta^{\prime}\right)-i \xi \Theta \\
& =(1-m) \xi\left(f^{\prime} \frac{\partial \Theta}{\partial \xi}-\theta^{\prime} \frac{\partial F}{\partial \xi}\right) .
\end{aligned}
$$

The dimensionless boundary conditions for (26)-(28) are

$$
\begin{aligned}
& F=F^{\prime}=0, H=-\frac{1}{2} F^{\prime \prime}, \Theta=1 \text { at } \eta=0, \\
& F^{\prime}=1, H=0, \Theta=0 \quad \text { as } \eta \rightarrow \infty .
\end{aligned}
$$

In the foregoing equation, $\xi=\omega x^{1-m} / U_{0}, G r_{x}=$ $g \beta\left(T_{w}-T_{\infty}\right) x^{3} / v^{2}$ is the Grashof number, $R e_{x}=U_{0} x^{m+1} / v$ is the Reynolds number, $R i=G r_{x} / R e_{x}^{2}$ is the Richardson's number, $\operatorname{Pr}=v / \alpha$ is Prandtl number, $K=\kappa / \mu$ is the vortex viscosity parameter and $M=\sigma B_{0}^{2} x^{1-m} /\left(\rho U_{0}\right)$ is the magnetic parameter.

The sets of equations (15)-(19) and (26)-(30) are solved by employing the straight forward finite difference method. Then we investigate the effects of the physical parameters on the skin friction, couple stress and the rate of heat transfer at the surface of the wedge. These are important not only from physical point of view but also from experimental point of view. In this study, the results will be presented in terms of the amplitudes of the skin friction, couple stress and the heat transfer rate having the following relations 


$$
\begin{aligned}
& A_{u}=\sqrt{F_{r}^{\prime \prime 2}+F_{i}^{\prime \prime 2}}, A_{m}=\sqrt{H_{r}^{\prime 2}+H_{i}^{\prime 2}}, \\
& A_{q}=\sqrt{\Theta_{r}^{\prime 2}+\Theta_{i}^{\prime 2}}
\end{aligned}
$$

respectively, where $\left(F_{r}^{\prime \prime}, F_{i}^{\prime \prime}\right),\left(H_{r}^{\prime}, H_{i}^{\prime}\right)$ and $\left(\Theta_{r}^{\prime}, \Theta_{i}^{\prime}\right)$ are the corresponding real and imaginary parts of the axial velocity, angular velocity and temperature gradients at the surface.

\section{Results and Discussion}

In this study, we have analyzed the mixed convection flow of micropolar fluid in presence of magnetic field. The governing equations have been solved by using the straight forward finite difference method for the entire frequency range. In order to get a clear insight on the physics of the problem, a parametric study is performed and the obtained numerical results are presented with the help of graphical illustrations.
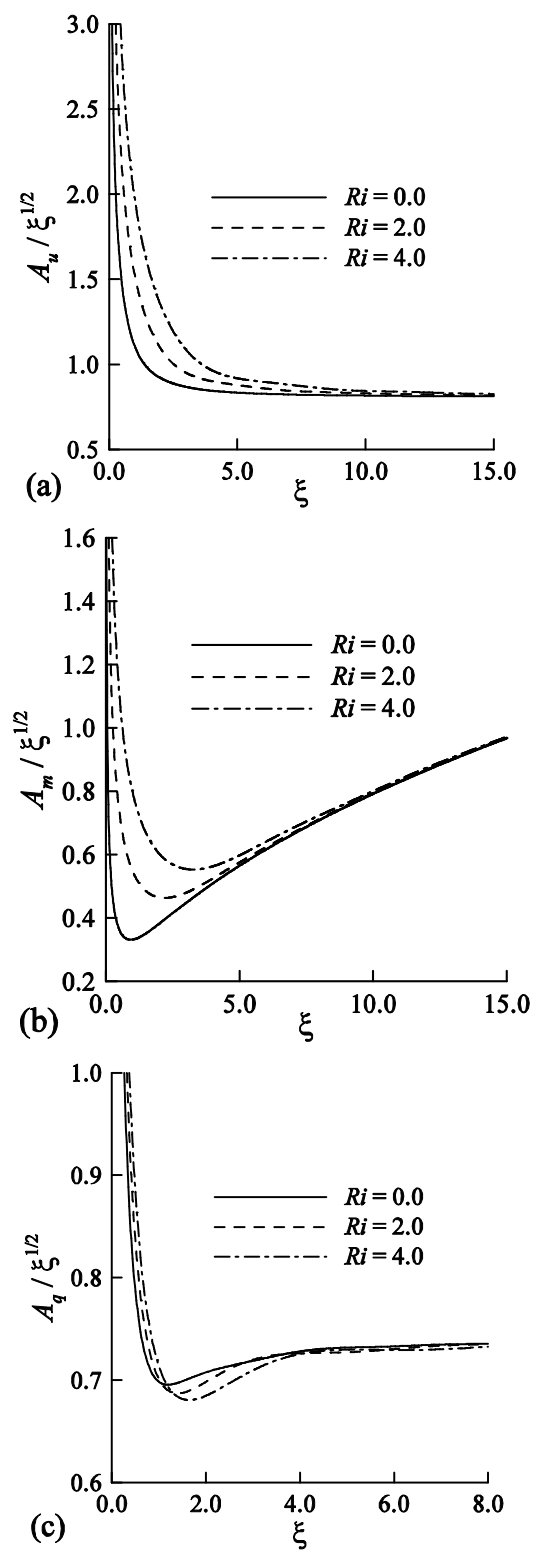

Fig. 2. Amplitudes of (a) skin friction, (b) couple stress and (c) heat transfer for different values of $R i$ while $M=0.2, \operatorname{Pr}=0.72$ and $K=2.0$.
The effects of the Richardson's number, $R i$, on the amplitudes of the skin friction, couple stress and heat transfer are shown in Figures 2(a), 2(b) and 2(c), respectively. From the figures, we observe that for higher Richardson's number, $R i$, the amplitudes of skin friction and couple stress are higher. On the other hand, for Richardson's number, $R i$, the amplitudes of heat transfer are higher near the leading edge while the reverse case is observed in the downstream region.
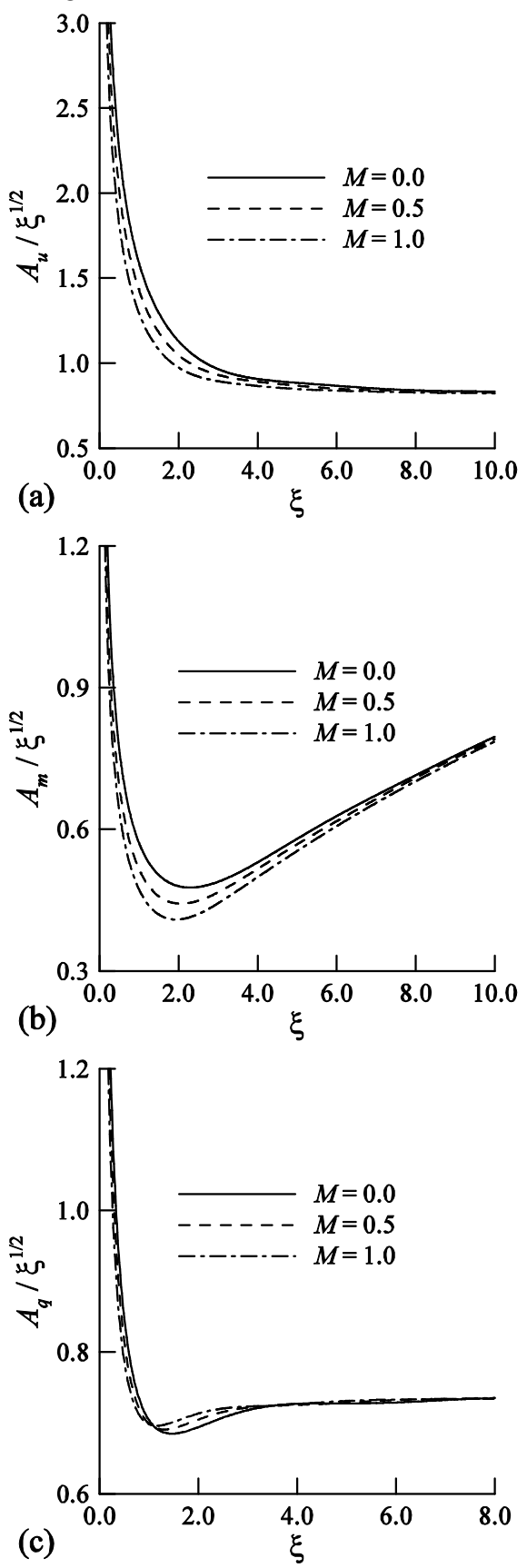

Fig. 3. Amplitudes of (a) skin friction, (b) couple stress and (c) heat transfer for different values of $M$ while $R i=2.0, \operatorname{Pr}=0.72$ and $K=2.0$.

Figures 3(a)-(c) illustrate the variations of the amplitudes of skin friction, couple stress and heat transfer, respectively, owing to the change of the magnetic parameter, $M$. With an increase of the magnetic parameter, the amplitudes of skin 
friction and couple stress decrease. From the definition of the magnetic parameter, it is evident that the magnetic parameter, $M$, increases owing to the increase of the strength of the magnetic field. As a result, the fluid velocity decreases with the increase in magnetic intensity. In other words, the imposition of the transverse magnetic field tends to retard the fluid flow. Thus the amplitudes of the skin friction and couple stress are lower for higher values of $M$. Besides, the amplitudes of heat transfer decrease near the leading edge owing to an increase of the magnetic parameter, $M$, while the reverse case is observed in the downstream region.
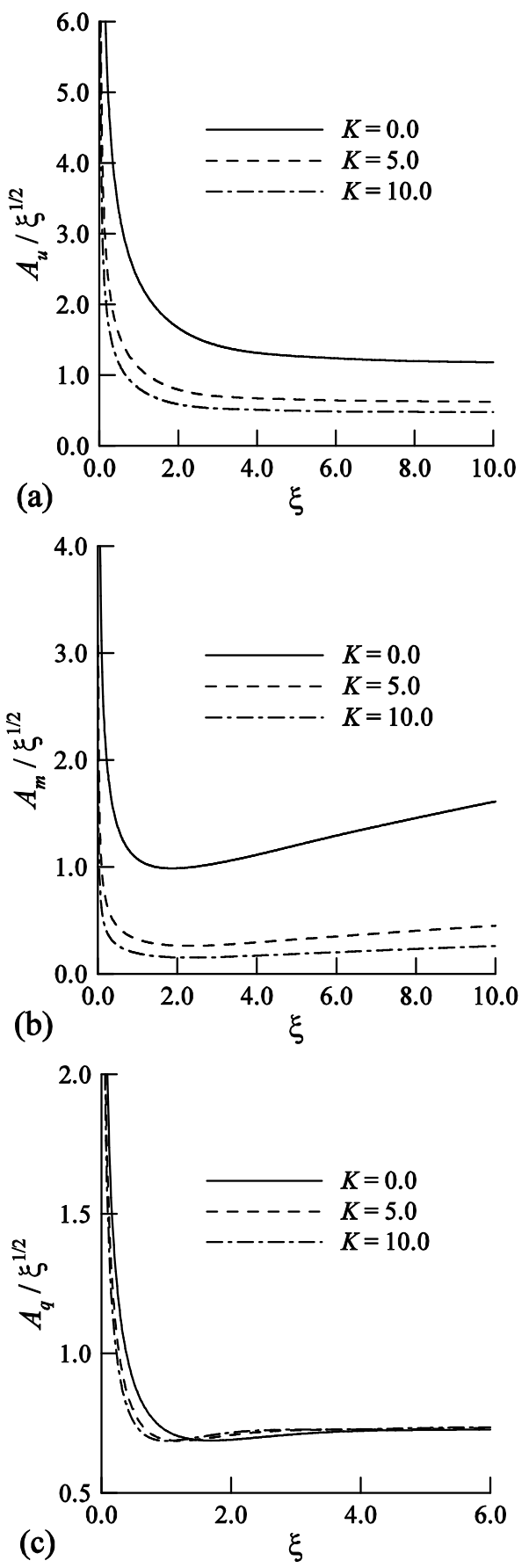

Fig. 4. Amplitudes of (a) skin friction, (b) couple stress and (c) heat transfer for different values of $K$ while $R i=2.0, \operatorname{Pr}=0.72$ and $M=0.2$.
The effects of the vortex viscosity parameter, $K$, on the amplitudes of skin friction, couple stress and heat transfer are shown in Figures 4(a)-(c), respectively. It is seen from the figures that when the vortex viscosity parameter, $K$, is increased, the amplitudes of skin friction and couple stress decrease. The definition of the vortex viscosity parameter $K$ $(=\kappa / \mu)$ indicates that the value of $K$ becomes higher either the coefficient of viscosity $\mu$ is lower or the coefficient of gyro-viscosity $\kappa$ is higher. This change of the fluid property reduces its velocity. Consequently, the amplitudes of skin friction and couple stress become lower for higher value of the vortex viscosity parameter, $K$. On the contrary, the amplitudes of heat transfer are found to be lower near the leading edge with an increase of $K$ and then the corresponding values of heat transfer become higher in the downstream region.

\section{Conclusions}

We examine the flow and heat transfer of a micropolar fluid over a vertical wedge due to oscillation in the free-stream and surface temperature. We found that the amplitudes of skin friction and couple stress strongly depend on the Richardson's number, $R i$, the magnetic parameter, $M$, and the vortex viscosity parameter, $K$. The amplitudes of skin friction and couple stress are higher for the Richardson's number, $R i$, while these become lower owing to the increase of the magnetic parameter, $M$, and the vortex viscosity parameter, $K$. Moreover, the effects of the parameters on the amplitudes of heat transfer are rather weak.

\section{References}

1. Eringen, A. C. 1966. Theory of micropolar fluids. J. Math. Mech., 16, 1-18.

2. Eringen, A. C. 1972. Theory of thermomicropolar fluids. J. Math. Anal. Appl., 38, 480-496.

3. Ariman, T. M. A. Turk and N. D. Sylvester, 1973. Application of microcontinum fluid mechanics. Int. J. Eng. Sci., 12, 273-293.

4. Willson, A. J., 1970. Boundary-layer in micropolar liquids. Proc. Camb. Phil. Soc., 67(2), 469-476.

5. Peddieson, J. and R. P. McNitt, 1970. Boundary layer theory for a micropolar fluid. Recent Adv. Eng. Sci., 5, 405-476.

6. Ahmadi, G. 1976. Self-similar solution of incompressible micropolar boundary layer flow over a semi-infinte plate. Int. J. Eng. Sci., 14, 639-646.

7. Ramachandran, N. T. S. Chen and B. F. Armaly, 1988. Mixed convection in stagnation flows adjacent to vertical surfaces. J. Heat Transf., 110(2), 373-377.

8. Gorla, R. S. R., 1976. Micropolar boundary layer flow at a stagnation point on a moving wall. Int. J. Eng. Sci., 14(7), 639-646.

9. Gorla, R. S. R., 1984. The axisymmetric micropolar boundary layer on a long thin cylinder. Int. J. Eng. Sci., 22, 293-299. 
10. Gorla, R. S. R., 1985. Axisymmetric thermal boundary layer of a micropolar fluid on a cylinder. Int. J. Eng. Sci., 23, p. 401-407.

11. Rees, D. A. S. and P. A. Bassom, 1996. The Blasius boundary-layer flow of a micropolar fluid, Int. J. Eng. Sci., 34, 113-124.

12. Ishak, A., R. Nazar, I. Pop, 2009. MHD boundary layer flow of a micropolar fluid past a wedge with constant wall heat flux. Commun. Nonlin. Sci. Numer. Simulat., 14, $109-118$.
13. Sacheti, N. C., P. Chandran and A. K. Singh, 1994. An exact solution for unsteady magnetohydrodynamic free convection flow with constant heat flux. Int. Commun. Heat Mass Transf., 21, 131-142.

14. Ibrahim, F. S., A. M. Elaiw and A. A. Bakr, 2008. Effect of the chemical reaction and radiation absorption on the unsteady MHD free convection flow past a semi infinite vertical permeable moving plate with heat source and suction. Commun. Nonlin. Sci. Numer. Simulat., 13, 1056-1066. 